\title{
Cloud Computing: A Solution to Information Support Systems (ISS)
}

\author{
Muzafar Ahmad Bhat \\ Department of Computer Sciences \\ University of Kashmir \\ $\mathrm{J} \& \mathrm{~K}$ India \\ Bashir Ahmad \\ Govt. S.P. Hr Sec. Institute \\ 190001- Srinagar \\ J \& K India
}

\author{
Razeef Mohd Shah \\ Department of Computer Sciences \\ University of Kashmir \\ $J \& K$ India
Inayat Rasool Bhat
SAM Degree College
Budgam - Kashmir \\ $\mathrm{J} \& \mathrm{~K}$ India
}

\begin{abstract}
Information Support Systems (ISS) are computer technology/network support systems that interactively support the information processing mechanisms for individuals and groups in life, public, and private organizations, and other entities. With the increasing use of technology in modern times, there is a growing requirement of Information Support Systems for the organizations.
\end{abstract}

Over some decades in the past, organizations have put efforts to be at the forefront of the development and application of computer-based Information Support Systems to collect, analyze and process the data and generate information to support decisions. Various computing paradigms have been employed for the purpose and needs have emerged for enormous infrastructure, unlimited system accessibility, cost effectiveness, increased storage, increased automation, flexibility, system mobility and shift of IT focus.

This paper presents a brief evaluation on how Cloud Computing paradigm can be used to meet the increasing demands of the Information Support Systems and how Cloud Computing paradigm can prove to be future solution for such systems.

\section{Keywords}

Cloud Computing, High Performance Computing, Microsoft's Windows Azure, Amazon Elastic Compute Cloud, Amazon Simple Storage Service, Service Oriented Architecture.

\section{INTRODUCTION}

Cloud Computing is evolving as a key computing platform for sharing resources that include infrastructures, software, applications, and business processes. Virtualization is a core technology for enabling cloud resource sharing [6].

Everyone has an opinion on what is Cloud Computing. It can be the ability to rent a server or a thousand servers and run a geophysical modeling application on the most powerful systems available anywhere [12].

Cloud computing [1][4] is an emerging trend to deploy and maintain software and is being adopted by the industry such as
Google, IBM, Microsoft, and Amazon. Several prototype applications and platforms, such as the IBM "Blue Cloud" infrastructure, the Google App Engine, the Amazon Cloud, and the Elastic Computing Platform [15]. Cloud Computing is perceived as the next progression that will impact organizational businesses and how they manage their IT infrastructures. The technology and architecture that cloud service and deployment models offer are a key area of research.

Even though there are numerous variations on the definition of Cloud Computing, some basic principles characterize this emerging computing paradigm. Cloud Computing provides technological capabilities - generally maintained off premisesthat are delivered on demand as a service via the Internet. Given that a third party owns and manages public cloud services, consumers of these services do not possess resources in the cloud model but pay for them on a per-use basis . Thus virtualization of the resources is the key concept [12]. In the real scenario, they are renting the physical infrastructure, platforms and applications within a shared architecture. Cloud offerings can vary from virtual infrastructure, computing platforms, centralized data centers to end-user Web-Services and Web applications to enormous other focused computing services.

Cloud Computing may be applied to solve problems in many domains of Information Technology like GIS (Geographical Information Systems), Scientific Research [2], e-Governance Systems [5], Decision Support Systems [15], ERP [4], Web Application Development [7], Mobile Technology [11] etc.

This paper briefly discusses the application of Cloud Computing as a computing paradigm to Information Support Systems (ISS) and how it can serve as a future technology for such systems.

\section{CLOUD COMPUTING: OVERVIEW}

According to NIST, National Institute of Standards and Technology, Cloud Computing is:

"Cloud Computing is a model for enabling convenient, ondemand network access to a shared pool of configurable computing resources (e.g., networks, servers, storage, applications, and services) that can be rapidly provisioned and 
released with minimal management effort or service provider interaction."

Cloud Computing is a general term for anything that involves delivering hosted services over the Internet. Instead of a static system architecture, Cloud Computing supports the ability to dynamically scale up and quickly scale down, offering cloud consumers high reliability, quick response times, and the flexibility to handle traffic fluctuations and demand [8]. Cloud Computing also supports multi tenancy, providing systems configured in such a way that they can be pooled to be shared by many organizations or individuals [3]. Virtualization technology allows cloud vendors to convert one server into many virtual machines, thereby eliminating client-server computing with single-purpose systems [4]. This maximizes hardware capacity and allows customers to leverage economies of scale [8].

\subsection{Cloud Computing Service Model}

The service model comprises three core options within the Cloud Computing environment.

\subsubsection{Software as a Service (SaaS)}

Sometimes referred to as "software on demand," is software that is deployed over the internet and/or is deployed to run behind a firewall on a local area network or personal computer. With SaaS, a provider licenses an application to customers either as a service on demand, through a subscription, in a "pay-as-you-go" model, or (increasingly) at no charge. This approach to application delivery is part of the utility computing model where all of the technology is in the "cloud" accessed over the Internet as a service. It is an approach whereby data is permanently stored in remote servers accessible via the Internet and cached temporarily on client devices that may include desktops, tablet computers, notebooks, hand-held devices, mobile phones, etc., and is frequently called Software as a Service (SaaS) [9]. The most commonly referenced examples of SaaS is Salesforce.com's human-resource applications, which provides a customer relationship management $(\mathrm{CRM})$ system accessible via the Internet Google Apps, Microsoft Office Live, Cisco WebEx ,Oracle CRM etc.

\subsubsection{Platform as a Service (PaaS)}

Perceived as Solution Stack as a service, provides an application platform, or middleware, as a service on which developers can build and deploy custom applications. PaaS offerings may include facilities for application design, application development, testing, deployment and hosting as well as application services such as team collaboration, web service integration and marshalling, database integration, security, scalability, storage, persistence, state management, application versioning, application instrumentation and developer community facilitation. These services may be provisioned as an integrated solution over the web. It facilitates the deployment of customercreated applications to the cloud using provider-supported frameworks, including APIs based on Java, Python, or .Net. Although the consumers don't control the underlying cloud infrastructure, they have control over the deployed applications and hosting environment configurations [9].

\subsubsection{Infrastructure as a Service (IaaS)}

Infrastructure as a Service is a stipulation model in which an organization outsources the equipment used to support operations, including storage, hardware, servers and networking mechanisms. When a vendor rents out infrastructure components on demand - such as servers, storage components, file systems, virtualization technologies, and network hardware-the vendor is delivering an IaaS service [10]. The service provider owns the equipment and is responsible for housing, running and maintaining it. The client typically pays on a per-use basis (Amazon Elastic Compute Cloud or Amazon Simple Storage Service e.g.).

A cloud service has three distinct characteristics that differentiate it from traditional hosting. It is sold on demand, typically by the minute or the hour; it is elastic -- a user can have as much or as little of a service as they want at any given time; and the service is fully managed by the provider (the consumer needs nothing but a personal computer and Internet access). Significant innovations in virtualization and distributed computing, as well as improved access to high-speed Internet and a weak economy, have accelerated interest in Cloud Computing [12].

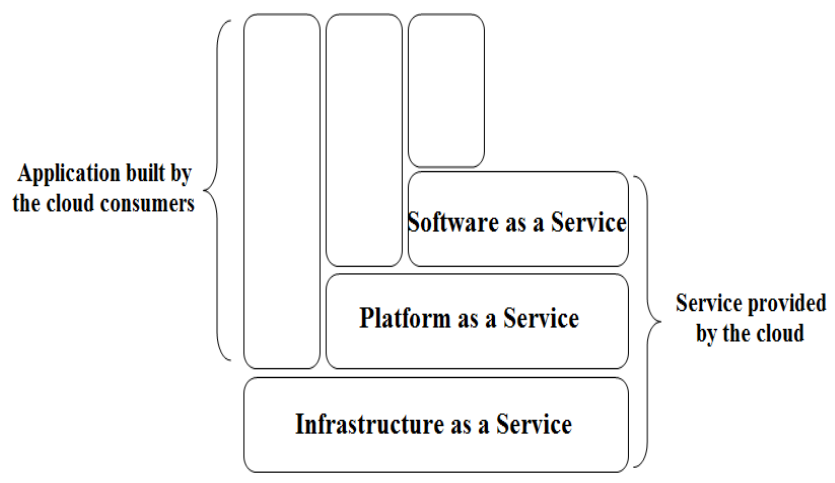

Figure 1: Cloud Computing Service Model

\subsection{Cloud Types}

In terms of implementation, there are three major types of cloud deployments; internal clouds, private clouds, and public clouds.

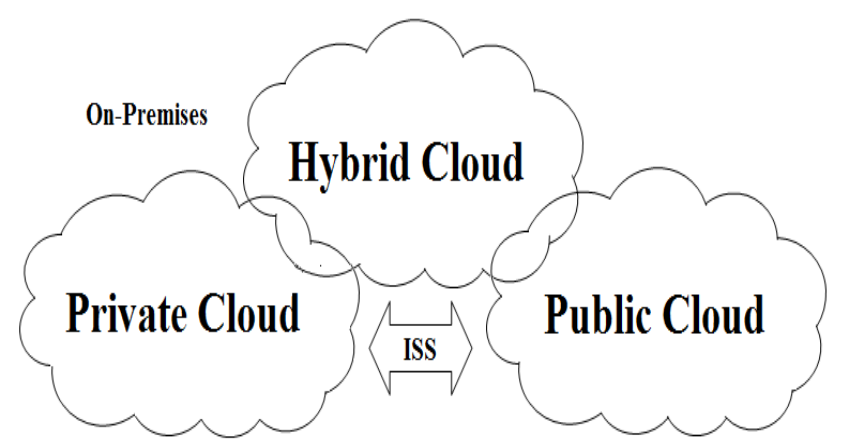

Figure 2: Cloud Types 


\subsubsection{Private Clouds}

Private clouds (aka, on-premises cloud) are cloud emplacements within the organization's location, managed internally in a private cloud-based service, data and processes are administered inside the organization by an IT department within an organization, without the limitations of network bandwidth, security exposures and legal requirements that using public cloud services might involve. In addition, private cloud services present the provider and the user greater control of the cloud infrastructure, improving security and adjustability because user access and the networks used are constrained and selected. This is growing as a new architecture for the Datacenter, sometimes known as a Datacenter-in-a-box. This model gives organizations a high level of control over the use of cloud resources while bringing in the expertise needed to establish and operate the environment [12].

\subsubsection{Public Clouds}

Public cloud services are characterized as being "off-premises" accessible to clients from a third party service provider via the Internet. Public clouds are most often hosted away from customer premises, and they provide a way to reduce customer risk and cost by providing a flexible, even temporary extension to enterprise infrastructure [12]. The name "public" does not always signify free, even though it can be free or to some extent economical to use. A public cloud does not mean that a user's data is publically available for all; public cloud vendors in fact offer an access control mechanism for their users. Public clouds provide an elastic, cost effective means to provide solutions. This type of cloud provides all the benefits of the economy of scale, ease of management, and ever growing elasticity.

\subsubsection{Hybrid Clouds}

In simpler terms hybrid cloud is a combination of a public and private cloud that interoperates. In this model users generally farm out no business- critical information and processing to the public cloud, while keeping business-critical services and data in their command. Hybrid Clouds are usually a combination of private clouds and public clouds, usually, managed using the same administration and monitoring consoles. A hybrid cloud also can be used to handle planned workload spikes. Sometimes called "surge computing," a public cloud can be used to perform periodic tasks that can be deployed easily on a public cloud [12].

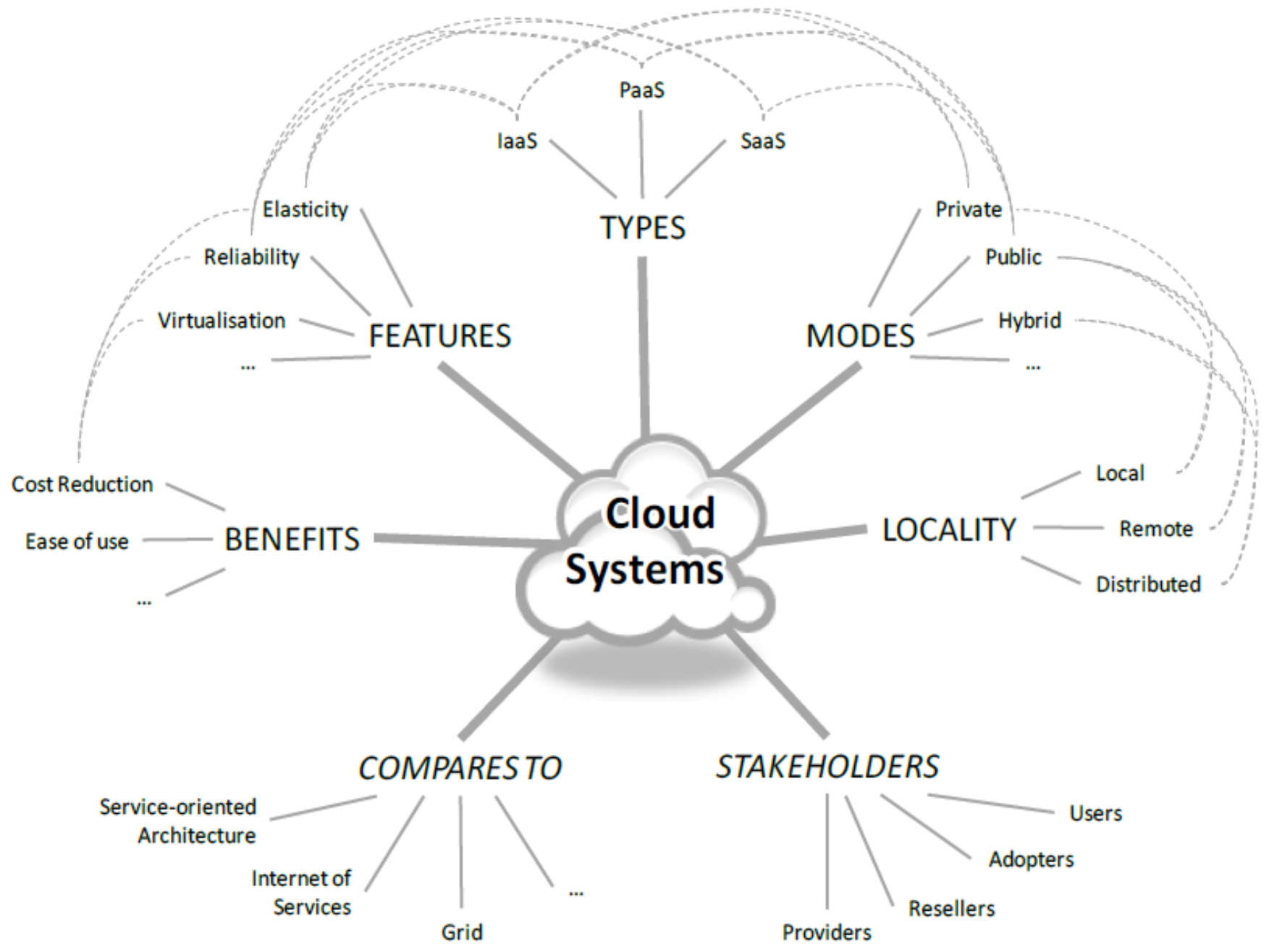

Figure 3: Non-Exhaustive View on the Main Aspects Forming a Cloud System [13] 


\section{INFORMATION SUPPORT SYSTEMS AND CLOUD COMPUTING}

Information Support Systems are computer based Information Systems that supports business or organizational information processing and information dissemination activities.

ISS serve the management, operations, and planning levels of an organization and provide information accessibility to a wide range of users distributed over a large geographical area.

ISS serves as the computer technology/network support system for varied users. ISS manages and provides technical support and service for centrally administered services such as software and hardware support. ISS is responsible for the upgrade and maintenance of both hardware and software for different shades of users of an organization/enterprise.

Over the past few years most of Information Support Systems are more and more dependent on high performance computing (HPC) environments such as clusters and computational grids [16]. Most of Information Support Systems usually deal with large volume of data (structured and unstructured) that requires huge CPU power to produce results in reasonable time on which wide range of users are dependent which may be located over a large geographical area. However, configuring and maintaining a cluster or a computational grid is usually a very cumbersome activity that requires specialists to support it. In addition, the high cost to acquire this computational apparatus can be considered a serious problem to the effective use of the ISS in terms of timeliness service and availability. Furthermore with the passage of time needs have emerged for enormous infrastructure, unlimited system accessibility, cost effectiveness, increased storage, increased automation, flexibility, system mobility and shift of IT focus.

Since Cloud Computing is a fast growing trend that includes several categories of service, all offered on demand over the Internet in a pay-as-you-go model, it promises to increase the velocity with which applications are deployed, increase innovation, and lower costs, all while increasing system agility. Using a Cloud Computing strategy for ISS will help in conducting core business activities with less hassle and greater efficiency. Organizations can maximize the use of their existing hardware to plan for and serve specific peaks in usage. Thousands of virtual machines and applications can be managed more easily using a cloud-like environment.

Businesses can also save on power costs as they reduce the number of servers required. And with IT staff spending less time managing and monitoring the data centre, IT teams are well placed to further streamline their operations as staff complete more work on fewer machines.

ISS in Cloud would refer to a collection of technologies that include hosted infrastructure (IaaS), like virtual servers, network equipment and disk space; hosted operating systems (PaaS), like Windows Azure and Google App Engine; and application-level services (SaaS).
The key characteristics of the Information Support Systems on cloud would be the ability to scale and provision computing power dynamically in a cost efficient way and the ability of the consumers (end users, organization or IT staff) to make the most of that power without having to manage the underlying complexity of the technology. The ISS Cloud architecture itself can be private (hosted within the organization's firewall) or public (hosted on the Internet). These characteristics lead to a set of core value propositions:

\subsection{On Demand Scalability}

The ISS have to deal with changes in their environments. The ability of Cloud Computing solutions to scale up and down is a major advantage. If an ISS has periods of time in which its computing resource needs are much higher or lower than normal, cloud technologies (both private and public) can cater to those changes. The ISS pays for the IT resources it actually uses; it does not have to maintain multiple sets of artificially high levels of resources to handle peak demands.

\subsection{Streamlining the Data Center}

ISS of any size will have a considerable investment in its data center. That includes buying and maintaining the hardware and software, providing the facilities in which the hardware is housed and hiring the personnel who keep the data center running inhouse. An ISS can streamline its data center by taking advantage of cloud technologies internally or by offloading workload into the public.

\subsection{Business Processes Improvement}

The cloud provides an infrastructure for improving business processes to a much greater extent. An ISS and its users, suppliers and partners can share data and applications in the cloud, allowing everyone involved to focus on the business process instead of the infrastructure that hosts it, thus minimizes the resource dependency.

\subsection{Minimizing Startup Costs}

For ISS that are just starting out, emerging markets, or even groups in larger organizations, Cloud Computing reduces startup costs to larger extent. The new ISS starts with an infrastructure already in place, so the time and other resources that would be spent on building a data center are borne by the cloud provider and taken care of, whether the cloud is private or public.

\section{CONCLUSION}

In this paper we discussed the Cloud Computing in general and discussed how Cloud Computing can improve ISS scenario. We also tried to highlight the benefits of using Cloud Computing Solution for ISS and how it can prove to be a future computing paradigm for Information Support Systems. Although it is an introductory and surface work we can conclude that Cloud Computing is a serious contender to aid Information Support Systems. We have not provided any particular architecture to be followed by Information Support Systems in cloud. 


\section{REFERENCES}

[1] Cloud Computing. Wikipdia. Available at http://en.wiki pedia.org/wiki/Cloud_computing

[2] Daniel Oliveira and Eduardo Ogasawara. Article: Is Cloud Computing the Solution for Brazilian Researchers?. International Journal of Computer Applications 6(8):19-23, September 2010.

[3] D. Oliveira, F. Baião, and M. Mattoso, 2010, "Towards Taxonomy for Cloud Computing from an e-Science Perspective", Cloud Computing: Principles, Systems and Applications (to be published), Heidelberg: Springer-Verlag.

[4] I. Foster, Y. Zhao, I. Raicu, and S. Lu, 2008, Cloud Computing and Grid Computing 360-Degree Compared, In: Grid Computing Environments Workshop, 2008. GCE '08, p. 10,1 .

[5] K.Mukherjee and G.Sahoo. Article: Cloud Computing: Future Framework for e-Governance. International Journal of Computer Applications 7(7):31-34, October.

[6] Liang-Jie Zhang, Qun Zhou, "CCOA: Cloud Computing Open Architecture," icws, pp.607-616, 2009 IEEE International Conference on Web Services, 2009.

[7] Liladhar R Rewatkar and Ujwal A Lanjewar. Article: Implementation of Cloud Computing on Web Application. International Journal of Computer Applications 2(8):28-32, June 2010. Published By Foundation of Computer Science.

[8] L.M. Vaquero, L. Rodero-Merino, J. Caceres, and M. Lindner, 2009, A break in the clouds: towards a cloud definition, SIGCOMM Comput. Commun. Rev., v. 39, n. 1, p. $50-55$.
[9] Rajarshi Chakraborty, Srilakshmi Ramireddy, T.S. Raghu, H. Raghav Rao, "The Information Assurance Practices of Cloud Computing Vendors", IT Pro July/August 2010, In IEEE Computer Society, p. 29-37.

[10] S. Bhardwaj, L. Jain, and S. Jain, "Cloud Computing: "Cloud Computing: A Study of Infrastructure as a Service (IaaS)," Int'l J. Eng. and Information Technology, vol. 2, no. 1, 2010, pp. 60-63.

[11] Sonal Anand, Sarvesh Gupta, Shweta Fatnani, Varsha Sharma and Deepti Jain. Article: Semantic Cloud for Mobile Technology. International Journal of Computer Applications 8(12):1-4, October 2010.

[12] Sun Microsystems White Paper, "Introduction to Cloud Computing Architecture,” June 2009.

[13] The Future of Cloud Computing, Opportunities for European Cloud Computing Beyond 2010. Expert Group Report. European Commission Information Society and Media, Public Version 1.0, pp 12.

[14] VOGELS, W. A Head in the Clouds - The Power of Infrastructure as a Service. In First workshop on Cloud Computing and in Applications (CCA '08) (October 2008).

[15] W.K. Chan, Lijun Mei, and Zhenyu Zhang, "Modeling and testing of cloud applications", to appear in Proceedings of 2009 IEEE Asia-Pacific Services Computing Conference (APSCC 2009), (Singapore, December 7-11, 2009), IEEE Computer Society Press, Los Alamitos, CA, USA, 2009.

[16] Y. Zhao, I. Raicu, and I. Foster, 2008, Scientific Workflow Systems for 21st Century, New Bottle or New Wine? In: 2008 IEEE Congress on Services, p. 467-471. 